\title{
BIG DATA: PAVING THE ROAD TO IMPROVED CUSTOMER SUPPORT EFFICIENCY
}

\author{
Ajay Parashar \\ Solution Architect, HiTech Industry Solution Unit, Tata Consultancy Services \\ ajay.parasharetcs.com
}

\begin{abstract}
The organizational adage 'customer is king' is not new. With a significant number of organizational resources devoted to understanding the 'king's' needs and responding to them, this phrase, in today's competitive business arena, is an understatement. With the increasing customer touch points and avenues for customers to provide formal/informal feedback, the modern day customer support ecosystem is a complex environment. There is a need to fuse the different components of support ecosystem to create a coherent system and Big Data platform is just the right catalyst that a flat-world organization today needs to re-energize its customer service effort and venture out to capture newer horizons. This white paper looks at the different components that make up the current customer support service environment and the challenges they pose to a uniform integration strategy. Finally it highlights how Big Data can be leveraged to achieve this strategy.
\end{abstract}

\section{KEYWORDS}

Big Data, Text Analytics, Speech Analytic, Customer Support.

\section{INTRODUCTION}

Steam engines triggered the beginning of the Industrial Age, and Henry Ford's Assembly Line kick-started a mechanical revolution that is still as dynamic as it was 100 years ago. In comparison, the IT revolution that can be pegged to start coinciding with Y2K boom is still in its infancy. Though we can communicate across the world in real-time, share photographs on our social networks instantly and receive comments on them even faster, an organization needs to cover several miles before it can develop a truly 360-degree understanding of the customer. Merely having a presence on social networks does not warrant a 360 degree customer understanding. These social networks need to be integrated with enterprise systems where the data received will besynthesized, processed and converted to meaningful insights that can help product design and development, or sales and marketing.

Organizations worldwide understand and acknowledge the fact that to be successful and remain competitive, it is important to have a healthy customer support ecosystem in place. While the

Sundarapandian et al. (Eds) : CCSEA, EMSA, DKMP, CLOUD, SEA - 2014

pp. 95-101, 2014. C CS \& IT-CSCP 2014

DOI : $10.5121 /$ csit.2014.4309 
customer may develop a preference for a company on account of the superior product quality initially, it is equally important to maintain an efficient customer support ecosystem to sustain the customer relationship in the long run and create a positive brand image. This support ecosystem is not merely limited to CRM systems, but should include every touch-point a customer can use to interact with the organization. Although organizations may have multiple IT systems managing customer information, it is rare to find them working in synergy. Even the capacity to indirectly influence sentiments is part of the broader customer service support ecosystem. An effective customer service ecosystem must clearly identify these disparate systems and get them to interact with each other in real-time. For example, if a call center executive has access to a sales representative's information sheet on a successful sale while addressing a customer enquiry, they may be able to tailor the response according to the problems reported to the Level 1/Level 2/Level 3 support desk for that particular model.

For HiTech industry characterized by a high rate of innovation, and hence, a high rate of obsolescence, it is imperative to have a very robust and fine-tuned support ecosystem in order to retain customers. A good example of this is the way the personal computer operating system space has evolved. It is not that Windows had a free run always; several equally competent operating systems came up from time to time like Linux. However, what kept customers linked with Windows as OS of choice, had a lot to do with the support ecosystem Microsoft was able to create behind it. They not only listened to the customer complaints and rectified them, but also from time to time kept evolving the OS to best address the increasing demand from perspective of a personal computer operating system, something that others could not do as effectively.

The situation that Microsoft faced and reacted to was not an isolated case, but a typical scenario that exists for all the participants of HiTech Industry segment. If we look across length and breadth of HiTech segment (be it a semiconductor manufacturers like Intel, Micron or Amat, or personal printer manufacturers like HP, Lexmark or Xerox) it is equally important for all to have a very robust support ecosystem to not only launch a successful product, but do it over and over without losing sight of customer expectation and reaction. Integrating the different components of the customer ecosystem such as marketing, sales, Level 1/Level 2 support desks, product research and development divisions is challenging due to their distinct purposes and the variety of data they generate. Big Data has the ability to handle and process variety of data, be it structured data from enterprise applications, unstructured text data from social media or semi-structured data from devices, under one platform. By enabling easy data integration, Big Data is slowly emerging as a key technology ingredient to overhaul customer support services.

\section{ALL ROADS LEAD TO CUSTOMER SUPPORT INTEGRATION}

For any organization, the product or services they offer go through a cycle of ideation, design, prototype, development, support, upgrade, and finally obsolescence. Similarly, from an organization's perspective, customer relationships go through a lifecycle involving identification, prospect development, prospect conversion, marketing approach, servicing, support, up-selling, cross-selling, retention and so on covered as part of Customer Life Cycle Management (CLM) process.

While this white paper references CLM to draw a high-level architecture of the customer service ecosystem, the focus here is largely on data and how data coming from different layers of CLM 
can be integrated to improve the efficiency of the entire customer support process. Figure 1 presents the different stages of CLM that are of interest from the customer support perspective.

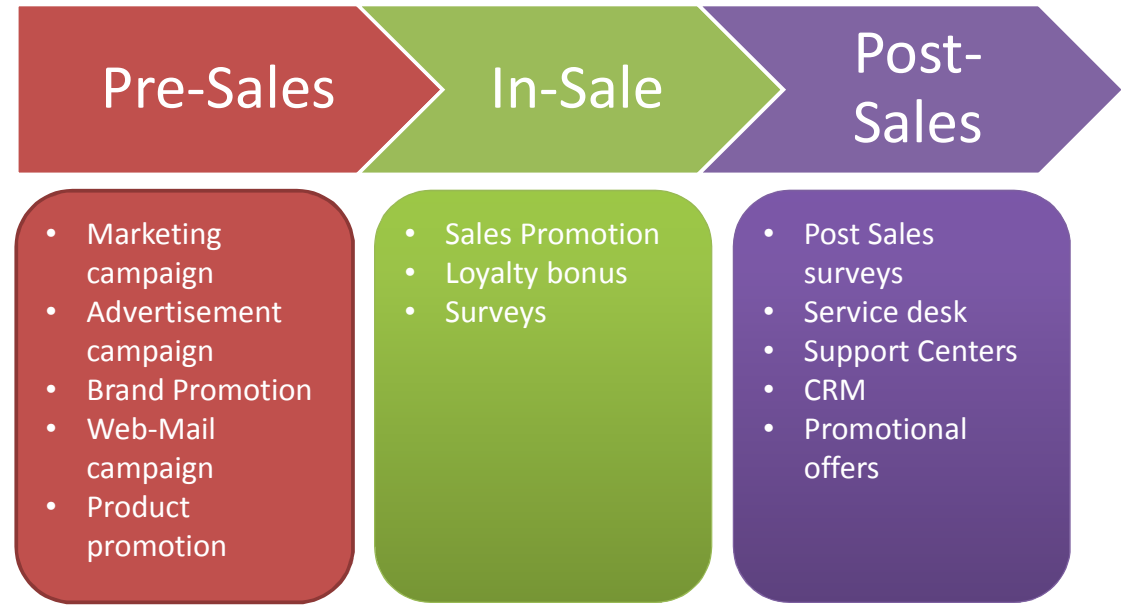

Figure 1: Customer Support stages

For a HiTech organization looking at selling enterprise software, the pre-sales stage will focus on identifying target organizations that may be interested in the enterprise solution to tailor marketing campaigns and promotional activities.

These different stages act in a way to support customers either by enabling them to make a decision or providing support post the decision process. There are several technology platforms that support the various customer support processes: tools to generate Web mail campaigns, enterprise level CRM suite, Interactive Voice Response (IVR) systems, tools to track customerreported issues, or systems to capture daily salesperson logs. Organizations, therefore, use a complicated set of systems - technologies and platforms, to provide seamless service to customers. However, integration between these systems is usually missing.

In the modern day ecosystem, social media has emerged as an important influence in the customers' decision making process. Before making a purchase, customers go through numerous reviews available on social channels, connect with friends and peers to seek opinions and carry out a comparative analysis between different products/services on offer. Therefore, an organization's success at converting a customer depends not only on the quality of the product/service it offers or the marketing campaign launched, but also on how the organization and its products are perceived by the wider community. This is where it is important for the organization to integrate its customer support ecosystem to develop a better understanding of customers and also provide them with unique post-sales support experiences.

The key to achievethis seamless integration lies in capturing, integrating, and analyzing the data generated at different customer touch-points, and making it available to the last line of customer support service. For example, in the current customer support landscape, it is almost impossible for a support engineer to establish a link between a customer support request, the advertisement campaign that triggered the buying process, and any product features highlighted by a sales representative that helped close the deal. 


\section{ROADBLOCKS TO DATA INTEGRATION}

Integration of systems that constitute the customer support ecosystem is encumbered by a multitude of factors that weigh against any effort made towards this integration. The primary impediments towards this integration are:

- Variety of data

- Variety of applications

- Information silos

Data: The biggest challenge that prevents organizations from integrating the different layers of customer engagement is the variety of data. Each layer of customer engagement has its own characteristics and generates data that is specific to that layer. For example, a front desk deals mainly with voice data while the Level 2/Level 3 support deals mostly with textual data with some attributes attached to it. Although data generated from pre-sales activities is structured, it does require further processing to be of use to the sales and post-sales layer.

Applications: The second challenge is the technology used to capture data across different layers. Typically, different applications are used for different layers of customer engagement and these applications work in isolation. In an organization, the marketing team makes use of digital marketing campaigns such asemail, social media channels or postal mails; the sales team uses enterprise planning systems for posting and tacking their orders; the customer support desk uses its own set of applications to interact with customers, monitor their service requests and raise tickets for issues that require more deeper analysis by the development team. Each of thesesystems supports a key organizational function or process. However, unified view of data residing in these isolated systems is lacking. Getting all these application systems to share data to create a truly integrated view is a difficult proposition from the conventional data management layer perspective.

Information silos: Today, organizations operate in multiple geographies and use different systems and tools depending on regional requirements. It is rare to find a single implementation of a CRM system across North America, Europe, Asia, and Africa. Regional factors, not restricted to language alone, play a crucial part in defining and creating an IT system that supports operations in a specific geography. One can debate endlessly on the futility of cross-geography information integration, but experience shows that cross-leveraging factors that support growth in one region may work equally well in other areas too.

Organizations realize the need to bring these information silos under one head and enable healthy collaboration, knowledge management, and uniform customer experience.

\section{DEFINING THE ROADMAP TO INTEGRATION}

In order to align itself with the changing market dynamics brought in by the increasing penetration of social channels in decision making process, it is important for organizations to create an environment that integrates the different customer touch points into a continuous flow of information that captures and transmits insights about a customer uniformly across the entire customer support ecosystem. 
Driving efficiency improvement through integration withthe application or the system level may not be cost-effective today, considering the varied mix of technologies used under customer support, as well as the geographic spread. Further, integrating applications that are custom built or from different vendors might not be a viable option. A data-centric approach then proves to be an effective mechanism for integration. The challenges posed by the variety of data components are effectively mitigated through proper utilization of newer technology elements that constitute the Big Data ecosystem. The right mix and proper orchestration of these newer technology elements provide a far more cost-effective mechanism to integrate the different layers of customer support services under a unified fabric.

A few technology paradigms that come under the spotlight include:

a) Textual data processing: Organizations, over a period of time, have collected huge amounts of pure text data such as entries in the 'Remarks' column of a feedback form, or the comments left by support executives in CRM systems. Earlier, due to technology constraints, it was difficult to process this data and convert it into actionable insights. However, today there are tools that work on the principles of Natural Language Processing (NLP) and efficiently churn out insights from this data. Presently, NLP is emerging as a front runner among the Big Data ecosystem technologies. Textual data can now be mined to understand the customer sentiment associated with a particular product family, the key features discussed by customers, and the changes over a period of time. Organizations can further integrate this information with data of their marketing campaigns carried out from time to time to understand how the campaign affected customer sentiment as well as the features highlighted through the campaign; an effective way to capture the return on investment (ROI) on the marketing campaign. There are several such instances where the capability to mine information from textual data has transformed relatively obscure data into an information goldmine.

b) Voice processing: The voice-based service/call center is a mainstay in the customer support strategy of any organization. It not only acts as the first point of contact between a customer and the organization, but also goes a long way in shaping an organization's brand perception. While organizations have invested heavily in setting up customer support desks or call centers, very little has been done to process the data captured from calls. Most organizations only rely on the efficiency of the support person to decipher the data and convert it into information.

Converting voice data into text whichcan be stored and analyzed further is a challenge that the technology world is experiencing today. There are several factors contributing to the challenges faced while deriving the right information from voice data. The tone of the caller, the accent, and the language used are among the most common ones. Voice processing or speech recognition is an area that is evolving rapidly. IBM's Watson participation and winning of the Jeopardy Quiz represents a step in the right direction to achieve complete speech capture, conversion, and usage. There are many more solutions like those from Dragon Naturally Speaking, TalkTyper, VoxSigma and others that are targeting the area of speech to text conversion and analysis.

c) Web Analytics: While aspects like voice to text conversion, text analytics and others have been the subject of research and used for some time now, Web analytics has gained traction only over the past few years. Albeit it is relatively new, the science of analyzing 
users' Web behavior has evolved tremendously. From using keywords on Web pages to render a more meaningful Web search which resultsincapturing every user activity on a Website, Web analytics has come a long way and found favor across organizations.

d) Structured enterprise data and unstructured data integration: Processing unstructured data (text or voice) on its own is not very significant unless the processed outcome is given as inputto the structured enterprise data to derive meaningful inferences. For example, an insightful picture of the sales performance will emerge only when the processed social media data is linked backed to the sales figures, and further enriched with information emerging from call center data.

Trying to integrate varied data sources on conventional relational database management platforms may not work due to their inherent inability to process data that does not fit into a structured, relational schema. Processing of text, voice, or data originating from devices (such as log files) requires an approach that is completely different from the number crunching approach that the relational databases are suited for. Platforms leveraging Big Data are emerging as platforms of choice for these kinds of operations. The recent global Big Data trend study survey conducted by TCS identifies customer support services as among the top three areas where companies worldwide are focusing their Big Data investments.

\section{CONCLUSION}

A well-tuned and integrated customer support and service infrastructure brings in additional benefits that add value to the organization. For instance, it brings greater transparency, better insights, and delivery of tailored services, leading to a prolonged and profitable engagement for both sides.

A comprehensive, integrated approach to customer support service can leverage Big Data to not only drive more efficient customer support, but also other areas within an organization such as product design. It is time to make the big move to Big Data!

\section{REFERENCES}

[1] Customer Life cycle management - What is it, How important it is http://www.salesboom.com/whitepapers/what_is_clm_whitepaper_summary.html

[2] Basics of Management - Customer Life Cycle Management http://basicsofmanagement.com/customer_life_cycle.php

[3] Transforming the lifetime of relationships between people and companies https://www-03.ibm.com/innovation/us/watson/

[4] Text Mining - http://en.wikipedia.org/wiki/Text_analytics 


\section{AUTHOR}

With a Master's degree in Business Administration and a Bachelor's degree in Industrial and Production Engineering, Ajay Parashar is a Solution Architect, and the Big Data lead for the TCS HiTech Industry Solution Unit.

Ajay has over 14 years of experience in database administration, project and program management, and delivery management. He has been extensively involved in business

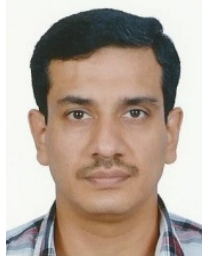
development projects around Big Data. 